\title{
Intervenção na prevenção e controle de câncer de colo uterino e mama numa unidade básica de saúde do nordeste do Brasil
}

\author{
Intervention on cervical and breast cancer prevention and control in a basic health unit in the \\ northeast of Brazil \\ Intervención en la prevención y control del cáncer de cuello uterino y mama en una unidad básica \\ de salud del nordeste de Brasil
}

Luis Sauchay Romeroa, Guilherme Barbosa Shimocomaquib, Ana Beatriz Rebêlo Medeirosc

\section{Resumo}

Trata-se de uma experiência realizada na unidade básica de saúde São Miguel II, Miguel Alves, Piauí, com o foco na prevenção e controle dos cânceres de colo de útero e de mama. Foram desenvolvidas ações em quatro eixos: organização e gestão, monitoramento e avaliação, engajamento público e qualificação da prática clínica. Após 12 semanas de intervenção, os indicadores de cobertura de atenção do câncer de colo de útero e do câncer de mama aumentaram, respectivamente, para $56,6 \%$ e $88,1 \%$. Já em relação aos indicadores de qualidade, $73,3 \%$ obtiveram amostras satisfatórias do exame colpocitológico de colo de útero, $73,3 \%$ e $23 \%$ mantiveram os registros adequados, respectivamente, das colpocitologias e das mamografias em registros específicos, 97,7\% das mulheres entre 25 e 64 anos foram avaliadas sobre sinais de alerta para câncer de colo e $98 \%$ das mulheres entre 50 e 69 anos foram avaliadas sobre risco para câncer de mama.

\section{Abstract}

This work is on an experience developed in the Sao Miguel II health unit, in Miguel Alves, state of Piaui, addressing the prevention and control of cervical and breast cancer. Actions were developed in four processes: organization and management, monitoring and assessment, public engagement and practical clinic qualification. After 12 weeks of intervention, coverage indicators of attention on cervical and breast cancer increased to $56.6 \%$ and $88.1 \%$, respectively. With respect to the quality indicators, $73.3 \%$ of satisfactory samples of pap smears were obtained, $73.3 \%$ of pap smears and $23 \%$ of mammographies were adequately registered in specific records, $97.7 \%$ of women aged between 25 and 64 were assessed on warning signs of cervical cancer and $98 \%$ of women aged between 50 and 69 were assessed on breast cancer risk.

\section{Resumen}

Se presenta una experiencia realizada en la unidad de salud São Miguel II, en Miguel Alves, Piauí, enfocada a la prevención y control de los cánceres de cuello uterino y mama. Se desarrolló en cuatro ejes temáticos: organización y gestión, seguimiento y evaluación, compromiso público y calificación de la práctica clínica. Después de 12 semanas de intervención, los indicadores de cobertura de atención al cáncer de cuello uterino y al cáncer de mama aumentaron para $56,6 \%$ y $88,1 \%$, respectivamente. Ya en relación a los indicadores de calidad, $73,3 \%$ obtuvieron las muestras satisfactorias del examen de Papanicolau, $73,3 \%$ y $23 \%$ mantuvieron los registros adecuados, respectivamente, de las citologías cervicovaginales y de las mamografías en registros específicos, así como 97,7\% de las mujeres entre 25 y 64 años fueron evaluadas sobre los señales de alerta para el cáncer de cuello uterino y $98 \%$ de las mujeres entre 50 y 69 años fueron evaluadas sobre el riesgo para el cáncer de mama.

Como citar: Romero LS, Shimocomaqui GB, Medeiros ABR. Intervenção na prevenção e controle de câncer de colo uterino e mama numa unidade básica de saúde do nordeste do Brasil. Rev Bras Med Fam Comunidade. 2017;12(39):1-9. http://dx.doi.org/10.5712/rbmfc12(39)1356
Palavras-chave: Atenção Primária à Saúde Saúde da Mulher Programas de Rastreamento Neoplasias do Colo do Útero Neoplasias da Mama

Keywords: Primary Health Care Women's Health Mass Screening Uterine Cervical Neoplasms Breast Neoplasms

Palabras clave: Atención Primaria de Salud Salud de la Mujer Tamizaje Masivo Neoplasias del Cuello Uterino Neoplasias de La Mama

Fonte de financiamento: declaram não haver. Parecer CEP: OF. 15/12 (UFPel), aprovado em 08/03/2012.

Conflito de interesses: declaram não haver. Procedência e revisão por pares: revisado por pares. Recebido em: 18/07/2016. Aprovado em: 16/08/2017. 


\section{Introdução}

A Organização Mundial da Saúde (OMS) aponta os cânceres de colo uterino e mama como os tipos mais frequentes na mulher no mundo e no Brasil. ${ }^{1} \mathrm{O}$ Instituto Nacional do Câncer (INCA) "José Alencar Gomes da Silva" estima que haverá 596.070 novos casos de câncer no Brasil no ano 2016, sendo os de pele, próstata e mama os mais incidentes.

Entre os homens, são esperados 295.200 novos casos e, entre as mulheres, 300.870. Entre as mulheres, as maiores incidências serão o câncer de mama (57.960), cólon e reto (17.620), colo do útero (16.340), pulmão (10.860), estômago (7.600), corpo do útero (6.950), ovário (6.150), glândula tireoide (5.870) e linfoma não Hodgkin (5.030). ${ }^{2}$ Desse modo, 11.190 novos casos de câncer de mama e 5.630 de câncer de colo de útero serão diagnosticados na região Nordeste do país, sendo que 580 de mama e 410 de colo de útero no estado de Piauí (PI). ${ }^{2}$

Estudos indicam aumento da sua incidência tanto nos países desenvolvidos quanto nos em desenvolvimento; ${ }^{3}$ assim, o câncer de colo de útero é o terceiro tumor mais frequente na população feminina, atrás do câncer de mama e do colorretal, e a quarta causa de morte de mulheres por câncer no Brasil. ${ }^{1}$ A causa principal desse último tipo é a infecção persistente por alguns tipos do Papilomavírus Humano (HPV), chamados também vírus oncogênicos. A infecção genital por este vírus é muito frequente e não causa doença na maioria das vezes. ${ }^{4}$

AAtenção Primária é o primeiro contato com o sistema de saúde e o local responsável pela organização do cuidado à saúde dos indivíduos, suas famílias e da população ao longo do tempo. É desenvolvida com alto grau de descentralização, capilaridade e próxima da vida das pessoas, ${ }^{5}$ que se justifica e propicia a implementação de estratégias efetivas de controle no nível comunitário orientadas à prevenção primária dessas doenças.

Assim, considerando a elevada morbimortalidade por câncer de mama e colo de útero, bem como as grandes possibilidades de promoção, prevenção, diagnóstico precoce e tratamento na atenção básica, o presente relato de experiência apresenta como objetivo melhorar a cobertura e a qualidade da atenção do programa de controle do câncer de colo de útero e de mama na área de abrangência da unidade básica de saúde São Miguel II, em Miguel Alves-PI.

\section{Relato da experiência}

O presente estudo faz parte do trabalho de conclusão de curso de especialização em saúde da família (maio 2015-abril 2016) de um profissional estrangeiro do Programa Mais Médico para o Brasil, na modalidade educação a distância (EaD), da Universidade Federal de Pelotas (UFPel), em parceria com a UNASUS, tendo sido aprovado pelo Comitê de Ética em Pesquisa dessa mesma instituição.

A experiência que será relatada consiste em uma intervenção desenvolvida por 12 semanas na área de abrangência da equipe de atenção básica (EAB) da Unidade Básica de Saúde (UBS) São Miguel II, por meio de ações de organização e gestão do serviço, engajamento público, qualificação da prática clínica e monitoramento e avaliação com o foco na prevenção e controle do câncer de colo de útero e de mama.

O município de Miguel Alves-PI, onde a UBS está localizada, possui uma população de $32.289,{ }^{6}$ sendo que $47 \%$ é do sexo feminino. A UBS se encontra localizada dentro do bairro São Miguel, na parte sul de 
Miguel Alves-PI (aproximadamente $110 \mathrm{~km}$ de Teresina, capital do estado), fundado como Vila em 24 de maio de 1912 com o nome em homenagem a seu fundador. Hoje com uma extensão territorial de 1.393,714km², tem $67 \%$ da população morando na zona rural. ${ }^{6}$ O SUS local compõe-se de 16 estabelecimentos de saúde administrados pela Prefeitura Municipal.

O foco da intervenção foi definido não apenas devido à verificação da baixa cobertura de atenção ao câncer de colo de útero (16\%) das mulheres entre 25 a 64 anos e ao câncer de mama (3\%) das mulheres de 50 a 69 anos, mas também por causa da não utilização de protocolos nacionais e as dificuldades da EAB quanto ao seu processo de trabalho.

No momento da intervenção, o número total da população cadastrada na UBS era de 2.641 , sendo 1.264 do sexo feminino. ${ }^{7}$ Destas, 636 e 238, respectivamente, são mulheres com idades entre 25 e 64 anos e 50 e 69 anos.

Para nortear e sustentar cientificamente a intervenção, utilizaram-se como referências os seguintes protocolos: Caderno da Atenção Básica № 13 - Controle dos cânceres do colo de útero e de mama do Departamento de Atenção Básica do Ministério da Saúde (2 edição, 2013), Diretrizes Brasileiras para o rastreamento do câncer do colo do útero (INCA, 2011) e Programa Nacional de Controle do Câncer de Mama (INCA, 2011).

Também foram utilizadas a planilha de objetivos (6), metas (13), indicadores (13) e ações para a elaboração e análise das ações da intervenção, assim como a planilha de coleta de dados (PCD) eletrônica e impressa para registro, armazenamento, monitoramento e avaliação da informação obtida de cada usuária. Essas planilhas que foram elaboradas e fornecidas pela UFPel.

Com o objetivo geral de melhorar a prevenção e o controle dos cânceres de colo de útero e de mama das mulheres residentes na área de abrangência da UBS São Miguel II, durante as 12 semanas de intervenção, foram estabelecidas metas de cobertura e de qualidade da atenção.

Quanto às primeiras, foram definidas em $50 \%$ as metas de cobertura da atenção ao câncer de colo de útero e de mama. Os critérios utilizados para essa definição basearam-se de acordo com as condições estruturais da unidade de saúde, a viabilidade, bem como o processo de trabalho da equipe. Já em relação às metas de qualidade, estas foram definidas em 100\%, considerando o princípio da integralidade da atenção.

Entre as metas de qualidade estabelecidas, encontram-se: obter $100 \%$ de coleta de amostras satisfatórias dos exames colpocitológicos; identificar $100 \%$ das mulheres com exame colpocitológico e mamografia alterados que não retornaram para conhecer o resultado; registrar $100 \%$ das mulheres atendidas; avaliar os sinais de alerta do câncer de colo de útero e de mama e orientar sobre doenças sexualmente transmissíveis (DST) e fatores de risco para o câncer de colo de útero e de mama em 100\% das mulheres atendidas. Todos esses objetivos e metas garantiram mapear as mulheres de risco nesses tipos de cânceres, e apoiar as atividades de promoção de saúde na área de abrangência.

De acordo com o projeto pedagógico do curso de especialização, e considerando os objetivos definidos previamente, planejaram-se o desenvolvimento de ações que englobaram quatro eixos temáticos básicos, avaliadores da qualidade da intervenção, e, por conseguinte, também do serviço da equipe no sentido da prevenção e o controle de ambos cânceres: monitoramento e avaliação, organização e gestão do serviço, engajamento público e qualificação da prática clínica (Quadro 1). 
Quadro 1. Ações desenvolvidas na intervenção, segundo eixos temáticos.

\begin{tabular}{|c|c|c|}
\hline Eixo temático & Ações & Controle/Profissional responsável \\
\hline \multirow{9}{*}{$\begin{array}{l}\text { Organização } \\
\text { e gestão do } \\
\text { serviço }\end{array}$} & 1. Estabelecimento do papel de cada profissional na ação programática. & Primeira semana/Médico \\
\hline & 2. Obtenção por meio do gestor de preservativos para distribuição na UBS. & Primeiras 4 semanas/Enfermeira \\
\hline & $\begin{array}{l}\text { 3. Divulgação na UBS de quadros e folders sobre prevenção de câncer de } \\
\text { mama. }\end{array}$ & $\begin{array}{l}\text { Primeiras } 4 \text { semanas/Técnica de } \\
\text { enfermagem }\end{array}$ \\
\hline & $\begin{array}{l}\text { 4. Acolhimento das usuárias de } 25 \text { a } 69 \text { anos de idade que demandem o serviço } \\
\text { de prevenção. }\end{array}$ & Permanente/EAB \\
\hline & 5. Cadastramento em planilha de coleta de dados. & Permanente/Médico \\
\hline & 6. Recebimento e registro dos resultados colpocitológico e mamografia. & Permanente/Técnica de Enfermagem \\
\hline & 7. Leitura individual dos resultados dos exames. & Permanente/Médico-Enfermeira \\
\hline & $\begin{array}{l}\text { 8. Encaminhamento ao nível secundário de atenção dos casos com resultados } \\
\text { positivos. }\end{array}$ & Permanente/Médico \\
\hline & 9. Elaboração de lista das usuárias faltosas. & Permanente/Médico-Enfermeira \\
\hline \multirow{8}{*}{$\begin{array}{l}\text { Monitoramento } \\
\text { e avaliação }\end{array}$} & 10. Verificação da adequabilidade das amostras colpocitológicas e mamografias. & Permanente/Médico-Enfermeira \\
\hline & 11. Avaliação individual dos resultados dos exames. & Permanente/Médico-Enfermeira \\
\hline & 12. Identificação das mulheres com maior risco de câncer. & Permanente/Médico-Enfermeira \\
\hline & $\begin{array}{l}\text { 13. Monitoramento da cobertura de detecção precoce de câncer, dos resultados } \\
\text { de todos os exames e do cumprimento e periodicidade. }\end{array}$ & Semana 4 e 12/Médico-Enfermeira \\
\hline & $\begin{array}{l}\text { 14. Monitoramento da avaliação de risco e do número de usuárias que } \\
\text { receberam orientações. }\end{array}$ & Semana 4 e 12/Médico-Enfermeira \\
\hline & 15. Monitoramento dos registros de todas as usuárias acompanhadas na UBS. & Semana 12/Médico-Enfermeira \\
\hline & 16. Monitoramento das usuárias faltosas. & A cada 4 semanas/Médico-Enfermeira \\
\hline & $\begin{array}{l}\text { 17. Monitoramento dos casos de prevenção de câncer de colo e câncer de } \\
\text { mama. }\end{array}$ & A cada 2 semanas/Médico-Enfermeira \\
\hline \multirow{6}{*}{$\begin{array}{l}\text { Engajamento } \\
\text { público }\end{array}$} & $\begin{array}{l}\text { 18. Esclarecimento e informação na comunidade sobre a importância } \\
\text { da realização, periodicidade, tempo de retorno e busca dos resultados } \\
\text { colpocitológicos e da mamografia }\end{array}$ & Permanente/ACS-resto da EAB \\
\hline & $\begin{array}{l}\text { 19. Esclarecimento e informação na comunidade sobre fatores de risco para } \\
\text { câncer de colo de útero e de mama. }\end{array}$ & Permanente/ACS-resto da EAB \\
\hline & $\begin{array}{l}\text { 20. Esclarecimento e informação na comunidade sobre seu direito de } \\
\text { manutenção dos registros de saúde no serviço, inclusive sobre a possibilidade } \\
\text { de solicitação de segunda via de opinião médica, se necessário. }\end{array}$ & Permanente/ACS-resto da EAB \\
\hline & $\begin{array}{l}\text { 21. Orientações à população sobre os sinais de alerta de câncer de colo de } \\
\text { útero e de mama e incentivo ao uso de preservativos. }\end{array}$ & Permanente/ACS-resto da EAB \\
\hline & $\begin{array}{l}\text { 22. Compartilhamento sobre a qualidade dos exames coletados e das condutas } \\
\text { esperadas para que possam exercer o controle social. }\end{array}$ & Permanente/EAB \\
\hline & $\begin{array}{l}\text { 23. Valorizar as sugestões da comunidade sobre estratégias para não ocorrer } \\
\text { evasão das mulheres. }\end{array}$ & Permanente/ACS-resto da EAB \\
\hline \multirow{4}{*}{$\begin{array}{l}\text { Qualificação da } \\
\text { prática clínica }\end{array}$} & $\begin{array}{l}\text { 24. Capacitação dos profissionais da equipe sobre o programa de prevenção, } \\
\text { fatores de risco, diagnóstico, tratamento e controle de câncer de colo de útero e } \\
\text { mama. }\end{array}$ & Primeira semana/Médico \\
\hline & 25. Capacitação dos profissionais da equipe sobre acolhimento. & Primeira semana/ Médico \\
\hline & $\begin{array}{l}\text { 26. Capacitação e treinamento dos profissionais da equipe sobre o sistema de } \\
\text { registros. }\end{array}$ & Primeira semana/ Médico \\
\hline & $\begin{array}{l}\text { 27. Disponibilizar protocolo técnico atualizado para consulta sobre o manejo dos } \\
\text { resultados dos exames. }\end{array}$ & Primeira semana/Médico-Gestor \\
\hline
\end{tabular}

ACS: agentes comunitários de saúde; EAB: equipe de atenção básica; UBS: unidade básica de saúde. 
É importante destacar que foram desenvolvidos momentos de educação permanente com a equipe antes de iniciar a intervenção, incorporando conhecimento atualizado do funcionamento dos programas de prevenção precoce, contribuindo com melhores resultados no trabalho comunitário dos agentes comunitários de saúde (ACS) da equipe de atenção básica e no acolhimento das usuárias.

Os momentos de educação permanente e a disponibilidade digital e impressa dos protocolos técnicos qualificou a prática clínica dos profissionais, além de possibilitar a reorganização do processo de trabalho e ampliou o escopo de ação, passos decisivos no avanço na integralidade da atenção na busca da qualidade, baseados nos princípios e diretrizes da Atenção Primária e do SUS.

Considerando que na UBS havia 636 mulheres com idades entre 25-64 anos, e que foi estabelecida uma meta de cobertura de atenção ao câncer de colo de útero de 50\%, o indicador de cobertura da atenção ao câncer de colo de útero atingido foi de 56,6\%, uma vez que, no final intervenção, 90 mulheres nessa faixa etária foram atendidas, das 159 projetadas nas 12 semanas de intervenção.

Já em relação ao indicador de cobertura de atenção ao câncer de mama, ao final da intervenção foi atingido 88,1\%, uma vez que na UBS havia 238 mulheres com idades entre 50-69 anos, e que foi estabelecida uma meta de cobertura também de $50 \%$, sendo que no final da intervenção 52 mulheres dessa faixa etária foram atendidas, das também 59 projetadas nas 12 semanas de intervenção.

Apenas 23\% (12) dessas mulheres realizaram mamografias em dia no final da intervação, porcentagem extremamente baixa, devido ao déficit de agendamentos na Secretaria Municipal de Saúde para a realização do exame em Teresina, onde três mamógrafos nas instituições que prestam serviço ao município estavam quebrados desde o mês anterior do início da intervenção, mantendo-se sem solução técnica até depois do encerramento.

Ademais, fatores econômicos também influenciaram de maneira significativa nesse resultado, pois era necessário efetuar o pagamento do serviço pela realização das mamografias. Este processo, informado e debatido oportunamente com o gestor, provocou dias de espera por agendamento e inúmeras idas e vindas das usuárias à Secretaria de Saúde pela procura do agendamento.

A percentagem de mulheres atendidas com amostras satisfatórias do exame colpocitológico foi de $73,3 \%$. Não foi possível alcançar essa meta devido ao fato de que até o final da intervenção os resultados finais dos exames colpocitológicos de 24 mulheres não tinham sido emitidos. Durante a intervenção, não houve mulheres com exames colpocitológicos alterados, assim, não foi necessário realizar a busca ativa.

Os indicadores relacionados aos registros adequados dos resultados dos exames colpocitológicos atingiram 73,3\% (66) das mulheres, levando-se em consideração apenas aquelas mulheres com exames e resultados completos, descartando-se as 24 mencionadas anteriormente pelo mesmo motivo.

As pesquisas de sinais de alertas para o câncer de colo de útero atingiram $97,7 \%$ (88) das mulheres. Esse mesmo resultado também foi alcançado para os indicadores de qualidade relacionados com a orientação sobre DST e fatores de riscos para o câncer de colo de útero. Apenas dois casos não foram pesquisados nem orientados devido à falha no atendimento da equipe ao começo da intervenção. 
Quanto aos registros adequados nos prontuários dos resultados das mamografias, de acordo com o Breast Image Reporting and Data System (BI-RADS), também são proporcionalmente baixos, devido aos fatores externos anteriormente mencionados, sendo igualmente de 12 casos. A avaliação do risco para o câncer de mama, as orientações sobre DST e fatores de riscos para este câncer tiveram resultados similares, sendo que 98\% (51) delas, não sendo erradamente avaliada 1 só usuária, considerado como pouco significativo, quase atingindo a meta projetada.

De maneira geral, embora sem atingir os resultados desejados, e visando abranger ao longo do ano um maior número de usuárias que não acessaram o atendimento, a intervenção proporcionou o aumento significativo da cobertura direcionada ao diagnóstico precoce dessas doenças, melhoria dos registros e qualificação da atenção. Indicadores específicos como exame de mamografia em dia, amostras satisfatórias de citologia e registro adequado de colpocitologia e mamografia que mostraram resultados mais baixos dependiam do retorno dos resultados das provas, fato que limitou o alcance.

Outras situações ou fatores interferentes internos foram a falta de comunicação de trabalho entre os membros da equipe no início da intervenção e as poucas atividades coletivas que se realizaram pela equipe na UBS. Apenas três ações de educação em saúde foram desenvolvidas durante os três meses de intervenção.

Entre as situações ou fatores externos que também interferiram negativamente, destacam-se a demora pelo gestor na entrega dos quadros e folders para divulgação das ações, a entrega dos preservativos para distribuição, a suspensão pelo gestor da ACS responsável pela maior microárea de abrangência da EAB a partir da terceira semana de desenvolvimento da intervenção, assim como também os ACS ficaram afastados durante duas semanas atuando no município no combate ao mosquito Aedes aegypti e na campanha de vacinação contra a raiva animal.

Além disso, observou-se um elevado intervalo de tempo (mais de 30 dias) para a obtenção dos resultados das colpocitologias com amostras tomadas, prolongando o tempo de espera das usuárias e fazendo-as voltarem à UBS várias vezes para conhecer os resultados.

Embora não tenha sido particularmente orientado no curso, focados na análise estratégica e elaboração do projeto de intervenção, aplicaram-se os princípios da análise DAFO, em função de identificar os principais problemas, limitações, dificuldades, fatores ou elementos negativos internos e externos. Esses elementos negativos internos mostraram as fragilidades da EAB na área escolhida. Também se identificaram fatores ou elementos positivos internos e externos (Quadro 2).

\section{Conclusão}

O processo de gestão para reduzir o risco de câncer de colo uterino e mama na área de abrangência da EAB da UBS São Miguel II se desenvolveu paralelamente aos princípios da atenção primária de saúde, que são inteiramente compatíveis.

Durante esse alinhamento e integração de ações concretas em função de fortalecer o processo da prevenção primária, a equipe estreitou ainda mais as relações com o gestor, a quem foi apresentado um relatório expondo os elementos considerados pendentes, expressados como negativos não apenas para a UBS, mas também para o sistema de saúde local. 
Quadro 2. Fatores internos e externos, limitadores e favorecedores no desenvolvimento do programa de prevenção e controle dos cânceres de colo de útero e mama na UBS São Miguel II.



ACS: agentes comunitários de saúde; EAB: equipe de atenção básica; INCA: Instituto Nacional do Câncer; OMS: Organização Mundial da Saúde; UBS: unidade básica de saúde; UFPel: Universidade Federal de Pelotas; * Fatores identificados durante a implementação da intervenção. 
As relações interpessoais e de trabalho da equipe igualmente se estreitaram, integraram-se às funções dos profissionais e agregaram-se às ações da intervenção à rotina de trabalho do serviço, priorizando na prática aquelas que ajudam a continuar ampliando com qualidade a cobertura e atenção. Da mesma maneira, fortaleceu o compromisso e engajamento público, aprendeu-se ainda mais a escutar, discutir em coletivo, pensar, refletir em equipe e definir pontos de vistas e perspectivas de trabalho, para alcançar metas e objetivos comuns, em conformidade com as políticas de saúde e necessidades do Brasil.

O impacto também se notou em atividades gerais do serviço tais como: acolhimento, organização e agendamento, controle e monitoramento. O serviço ganhou em qualidade de atenção com as ações de qualificação da prática clínica e na implantação de estratégias de monitoramento e avaliação que se fizeram periodicamente, avançando-se na concretização de avaliações, registros e agendamentos, reajustando as condutas nas consultas médicas e de enfermagem com novos arranjos de trabalho, assim como viabilizando a otimização da agenda para a atenção a demanda espontânea.

O trabalho comunitário dos ACS foi outro elemento que ficou fortalecido ao se estabelecer métodos e objetivos de trabalho mais intencionalmente direcionados, permitindo mapear as mulheres de maior risco, e ao mesmo tempo ensinar e compartilhar com os seus membros múltiplas informações sobre o foco da intervenção.

A combinação desses aspectos na comunidade e a ampliação do seu conhecimento sobre periodicidade, sistematicidade, tempo de retorno para busca dos resultados colpocitológicos e da mamografia, fatores de risco, sinais de alerta e sobre seu direito de manutenção dos registros de saúde no serviço, inclusive sobre a possibilidade de solicitação de segunda via de opinião médica se necessário, facilitaram o exercício de controle social das usuárias atingidas.

Isso demostrou a ampliação da conscientização e valorização das mulheres da área de abrangência, respeito aos seus problemas de saúde, o que pouco a pouco iria-se reproduzir entre elas como o efeito da bola de neve, potencializado pela estabilidade e orientação da EAB.

A intervenção como parte do curso de especialização também constituiu uma grande experiência no tipo de aprendizado para o autor principal deste trabalho, particularmente pelo formato de ensino a distância. Os momentos de qualificação da prática clínica e engajamento público recebido por meio do desenvolvimento do curso, impulsionou o conhecimento sobre o SUS, necessário para desenvolver um melhor trabalho nele, e também ampliou os conhecimentos na área da prática da medicina de família e comunidade no Brasil.

Finalmente, em relação aos resultados concretos deste trabalho, a EAB enxergou novas e atualizadas perspectivas de trabalho, pretendendo de antemão viabilizar os seus esforços em direção ao desenvolvimento de ações programáticas nos programas de atenção ao pré-natal e puerpério, saúde da criança, atenção ao idoso, hipertensão arterial e diabetes mellitus.

\section{Referências}

1. Ministério da Saúde. Instituto Nacional do Câncer. INCA e Ministério da Saúde apresentam estimativas de câncer para 2014. Rio de Janeiro: INCA; 2014. [acesso 2016 Jul 5]. Disponível em: http://www2.inca.gov.br/wps/wcm/connect/agencianoticias/site/home/noticias/2013/ inca_ministerio_saude_apresentam_estimativas_cancer_2014

2. Ministério da Saúde. Instituto Nacional de Câncer José Alencar Gomes da Silva. Coordenação de Prevenção e Vigilância. Estimativa 2016: incidência de câncer no Brasil. Rio de Janeiro: INCA; 2015. 
3. Ministério da Saúde. Instituto Nacional do Câncer. Tipos de câncer: Mama. Rio de Janeiro: Ministério da Saúde. [acesso 2016 Jul 5]. Disponível em: http://www2.inca.gov.br/wps/wcm/connect/tiposdecancer/site/home/mama

4. Ministério da Saúde. Instituto Nacional do Câncer. Tipos de câncer: Colo de útero. Rio de Janeiro: Ministério da Saúde. [acesso 2016 Jul 5]. Disponível em: http://www2.inca.gov.br/wps/wcm/connect/tiposdecancer/site/home/colo_utero

5. Portal da saúde SUS. Tipos de câncer: PNAB - Política Nacional de Atenção Básica. Brasília: Departamento de Atenção Básica. [acesso 2016 Jul 5]. Disponível em: http://dab.saude.gov.br/portaldab/pnab.php

6. Brasil. Instituto Brasileiro de Geografia e Estatística. [Internet]. Piauí. Miguel Alves; [acesso 2016 Jul 5]. Disponível em: https://cidades. ibge.gov.br/v4/brasil/pi/miguel-alves/panorama

7. Brasil. Instituto Brasileiro de Geografia e Estatística. Piauí. Miguel Alves 2016. [Internet]. [acesso 2016 Jul 5]. Disponível em: http://cidades. ibge.gov.br/xtras/temas.php?lang=\&codmun=220620\&idtema=16\&search=|ls\%EDntese-das-informa\%E7\%F5es

a Programa Mais Médicos para o Brasil; Equipe de Atenção Básica São Miguel II. Miguel Alves, PI, Brasil. lusaurom@gmail.com

b Universidade do Vale do Rio dos Sinos (UNISINOS). São Leopoldo, RS, Brasil. g.shimocomaqui@gmail.com (Autor correspondente)

` Equipe de Atenção Básica São Miguel II. Miguel Alves, PI, Brasil. anabeatriz6996@gmail.com 